\title{
Pairings of $p$-Compact Groups and $H$-Structures on the Classifying Spaces of Finite Loop Spaces
}

\author{
Dedicated to Professor Fuichi Uchida on his 60th birthday
}

By

\author{
Kenshi IsHIGURO*
}

\begin{abstract}
We consider the maps between classifying spaces of $p$-compact groups of the form $B X \times B Y \rightarrow B Z$. The main theorem shows that if the restriction map on $B Y$ is a weak epimorphism, then the restriction on $B X$ should factor through the classifying spaces of the center of the $p$-compact group $Z$.
\end{abstract}

\section{Introduction}

In [8], the author investigated certain pairing problems for classifying spaces of compact Lie groups. The main work in this paper can be regarded as a $p$-compact group version. Dwyer-Wilkerson [3] defined a $p$-compact group and studied its properties. The purely homotopy theoretic object appears to be a good generalization of a compact Lie group at the prime $p$. A $p$-compact group has rich structure, such as a maximal torus, a Weyl group, etc. A note of Møller [13] summarizes their work. Further development on the homotopy theory of $p$-compact groups can be seen, for example, in [4], [14], [15], [2] and [18]. We first recall some basic things about the $p$-compact groups and pairing problems, and then state our main results.

A $p$-compact group, [3], is a loop space $X$ such that $X$ is $F_{p}$-finite and that its classifying space $B X$ is $F_{p}$-complete. The $p$-completion of a compact Lie group $G$ is a $p$-compact group if $\pi_{0}(G)$ is a $p$-group. For an odd dimensional

Communicated by K. Saito, February 3, 1998.

1991 Mathematics Subject Classification(s): 55R35, 55P15, 55P60.

* Fukuoka University, Fukuoka 814 0180, Japan. 
sphere $S^{2 n-1}$, it is known that its $p$-completion has a loop structure if $n$ divides $p-1$. This is an example of $p$-compact groups other than compact Lie groups. More examples are known as Clark-Ewing $p$-compact groups, [13, §2].

For $p$-compact groups $X$ and $Y$, a pointed map $f: B X \rightarrow B Y$ is called a homomorphism. Let $Y / X$ denote the homotopy fibre of $f$. The homomorphism $f$ is called a monomorphism if $Y / X$ is $F_{p}$-finite, and an epimorphism if the loop space $\Omega(Y / X)$ is a $p$-compact group.

The centralizer of $f$ is loop space of the component containing $f$ of the mapping space of unpointed maps, denoted by $\Omega \operatorname{map}(B X, B Y)_{f}$. A homomorphism is called central if the evaluation map, $e v: \operatorname{map}(B X, B Y)_{f} \rightarrow B Y$, is a homotopy equivalence. According to [4], any $p$-compact group $X$ has a unique maximal central subgroups that is called the center of $X$ and denoted by $C(X)$. It is also shown in [4] that $B C(X) \simeq \operatorname{map}(B X, B X)_{i d}$ where $i d: B X \rightarrow B X$ is the identity homomorphism.

Next we recall pairing problems for $p$-compact groups and compact Lie groups, [8] and [17]. Suppose that $X, Y$ and $Z$ are $p$-compact groups, and that $\alpha: B X \rightarrow B Z$ and $f: B Y \rightarrow B Z$ are homomorphisms. The homotopy class of $\alpha$ is said to be contained in the set of the homotopy classes of axes $f^{\perp}(B X, B Z)$ if there is a map (called a pairing) $\mu: B X \times B Y \rightarrow B Z$ with restrictions (axes) $\left.\mu\right|_{B X} \simeq \alpha$ and $\left.\mu\right|_{B Y} \simeq f$. In other words, if $\alpha \in f^{\perp}(B X, B Z)$, we have the following homotopy commutative diagram:

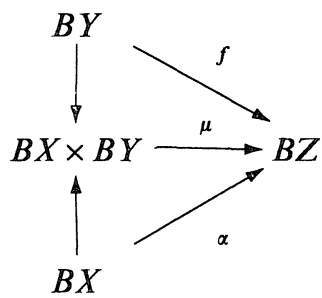

We note that $f^{\perp}(B X, B Z)$ is a subset of the homotopy set $[B X, B Z]$. For a weak epimorphism $f$ of the classifying spaces of connected compact Lie groups, the set of homotopy classes of axes has been determined in [8]. In this paper we will obtain analogous results for $p$-compact groups.

In [9], for connected compact Lie groups $L$ and $G$, a map $B L \rightarrow B G$ or $B L_{p}^{\wedge} \rightarrow B G_{p}^{\wedge}$ is called a weak epimorphism, if there exists a fibration $F \rightarrow B L \rightarrow B G$ or $F \rightarrow B L_{p}^{\wedge} \rightarrow B G_{p}^{\wedge}$ such that $H^{*}(\Omega F ; Q)$ is a finite dimensional $Q$-module or 
that $H^{*}\left(\Omega F ; Z_{p}^{\wedge}\right) \otimes Q$ is a finite dimensional $Q_{p}^{\wedge}$-module, respectively. The second condition of the following theorem requires a similar assumption for a homomorphism of connected $p$-compact groups $f: B Y \rightarrow B Z$. By the way, the connectivity is not assumed in the first condition. The result below is a generalization of $[8$, Theorem 1$]$.

Theorem 1. Suppose $X$ is a p-compact group. If either

(i) $f: B Y \rightarrow B Z$ is an epimorphism of p-compact groups, or

(ii) $f: B Y \rightarrow B Z$ is a homomorphism of connected p-compact groups such that $H^{*}\left(\Omega(Z / Y) ; Z_{p}^{\wedge}\right) \otimes Q$ is a finite dimensional $\boldsymbol{Q}_{p}^{\wedge}$-vector space

then the following hold:

(1) If $\alpha \in f^{\perp}(B X, B Z)$, then the map $\alpha$ factors through the classifying space of the center of $Z$, denoted by $C(Z)$, up to homotopy.

(2) Moreover, we have $f^{\perp}(B X, B Z)=[B X, B C(Z)]$.

If the mapping space $\operatorname{map}(B Y, B Z)_{f}$ is homotopy equivalent to $B C(Z)$, the proof can be immediate. This is the case under the assumption (i). A result of Dwyer-Wilkerson [4, Lemma 10.3] implies $\operatorname{map}(B Y, B Z)_{f} \simeq B C(Z)$ if $f: B Y \rightarrow B Z$ is an epimorphism. Our proof of Theorem 1, however, doesn't rely on the precise recognition of the mapping space. Under the two different assumptions, the arguments go parallel. Since there may be independent interest, a portion of the argument under the assumption (i) is included.

Here we make a remark analogous to the one in [8]. Taking $Y=Z$ and $f=i d$, our problem asks possible $B X$-actions on $B Y$. A consequence of Theorem 1 shows that such an action under $\alpha$ exists if and only if the orbit map $\alpha: B X \rightarrow B Y$ is central. We see, for instance, that there are no nontrivial $B X$-actions on $B\left(S^{2 n-1}\right)_{p}^{\wedge}$ for $n \geq 3$, since the center $C\left(\left(S^{2 n-1}\right)_{p}^{\wedge}\right)$ is contractible.

A connected $p$-compact group $Y$ is called semi-simple if $\pi_{1}(Y)$ is finite, [14]. In this case, the center $C(Y)$ is a finite abelian $p$-group, [15]. If $X$ is connected and $Y$ is semi-simple, the homotopy set $[B X, B C(Y)]$ is trivial. Consequently, there are likewise no nontrivial $B X$-actions on $B Y$.

Furthermore, if we take $X=Y=Z$ and $f=\alpha=i d$, the problem now asks whether $B X$ is an $\mathrm{H}$-space. A pairing $\mu: B X \times B X \rightarrow B X$ could be the 
$H$-multiplication. Before stating our result, recall that a $p$-compact group $X$ is called abelian if $e v: \operatorname{map}(B X, B X)_{i d} \rightarrow B X$ is a equivalence. Any abelian $p$-compact group is equivalent to the product of a $p$-compact torus and a finite abelian $p$-group, [4] and [15]. Corollary 2 stated in $\S 2$ implies that $B X$ is an $\mathrm{H}$-space if and only if $X$ is abelian. This result holds when a $p$-compact group $X$ is replaced by a finite loop space.

Theorem 2. Suppose $X$ is a finite loop space. If its classifying space $B X$ is an $\mathrm{H}$-space, then $X$ is equivalent to the product of a torus and a finite abelian group.

The above result is a generalization of Corollary 2.4 in [8]: If $G$ is a compact Lie group and $B G$ is an $\mathrm{H}$-space, then $G$ is an abelian group. Theorem 3 in $\$ 2$ will give the $p$-completed version of this result. Namely, if $(B G)_{p}$ is an $\mathbb{H}$-space, then $G$ is $p$-nilpotent in the sense of [6]. The group $G$ need not be abelian. We can find, however, an abelian compact Lie group $A$ such that $(B G)_{p}^{\wedge} \simeq B A$.

The author would like to thank Chuck McGibbon for his comments.

\section{§1. Mapping Spaces and Proof of Theorem 1}

We will prove Theorem 1 in this section. To do so, we need a few basic results about $p$-compact groups. The following lemma translates a setting of groups to a homotopy setting of $p$-compact groups.

Lemma 1. Suppose $j: B X \rightarrow B Y$ and $q: B Y \rightarrow B Z$ are homomorphisms of p-compact groups. If the composite map $q \cdot j$ is a homotopy equivalence (isomorphism), then $j$ is a monomorphism and $q$ is an epimorphism.

Sketch of Proof. We sketch the proof. From our assumption, one can show that $Y \simeq \Omega(Z / Y) \times Z$ and $\Omega(Z / Y) \simeq Y / X$. Thus $Y / X$ is $\mathbb{F}_{p}$-finite, and $\Omega(Z / Y)$ is a $p$-compact group. Therefore $j$ is a monomorphism and $q$ is an epimorphism.

We recall [3, Theorem 9.7] that if a $p$-compact group $X$ is connected, the cohomology algebra $H^{*}\left(B X ; \mathbb{Z}_{p}^{\wedge}\right) \otimes Q$ is a polynomial ring over $Q_{p}^{\wedge}$ 
concentrated in even degree. The number of the generators of the polynomial algebra is called rank of $X$ and denoted by $\operatorname{rank}(X)$. If $n=\operatorname{rank}(X)$, it is known that the maximal torus of $X$ is equivalent to $\left(B T^{n}\right)_{p}^{\wedge}$. It is also known that $H^{*}\left(B X ; Z_{p}^{\wedge}\right) \otimes Q$ is isomorphic to the invariant $\operatorname{ring}\left(H^{*}\left(B T^{n} ; Z_{p}^{\wedge}\right) \otimes Q\right)^{W(X)}$, where $W(X)$ is the Weyl group of $X$.

\section{Proposition 1. Suppose either}

(i) $X, Y$ and $Z$ are p-compact groups, $i: B X \rightarrow B Z$ is a monomorphism and $f: B Y \rightarrow B Z$ is an epimorphism, or

(ii) $X, Y$ and $Z$ are connected p-compact groups, $i: B X \rightarrow B Z$ is a monomorpism and $f: B Y \rightarrow B Z$ is a homomorphism such that $H^{*}\left(\Omega(Z / Y) ; Z_{p}^{\wedge}\right) \otimes Q$ is a finite dimensional $\boldsymbol{Q}_{p}^{\wedge}$-vector space.

If there is a map (extension) $\tilde{f}: B Y \rightarrow B X$ with $f \simeq i \cdot \tilde{f}$,

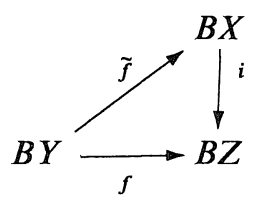

then $B X$ is equivalent to $B Z$ under the map $i$.

Proof. First assume the condition (i). It suffices to show that $i: B X \rightarrow B Z$ is an epimorphism. Recall that $f: B Y \rightarrow B Z$ lifts to $\tilde{f}$ if and only if the homotopy fixed point $(Z / X)^{h Y}$ is nonempty, [3, §3.3]. Since $f: B Y \rightarrow B Z$ is an epimorphism, by definition, the loop space $\Omega(Z / Y)$ is a $p$-compact group. Let $U=\Omega(Z / Y)$ so that $B U \rightarrow B Y \rightarrow B Z$ is a fibration of $p$-compact groups. Then $(Z / X)^{h Y}$ is homotopy equivalent to $\left((Z / X)^{h U}\right)^{h Z}$. Notice here that the action of $U$ on $Z / X$ is trivial. Since the Sullivan conjecture for $p$-compact groups holds, [4, Theorem 9.3], we see $(Z / X)^{h U} \simeq Z / X$. Consequently $(Z / X)^{h Y} \simeq(Z / X)^{h Z}$. This means that $(Z / X)^{h Z}$ is nonempty, and therefore the identity map $1_{B Z}: B Z \rightarrow B Z$ lifts to a map $r: B Z \rightarrow B X$ so that $i \cdot r \simeq 1_{B Z}$.

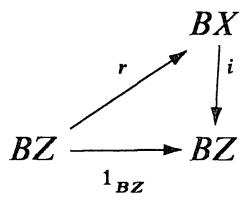


From Lemma 1 the monomorphism $i$ is also an epimorphism. Hence $i$ is an isomorphism.

Next assume the condition (ii). Since $H^{*}\left(\Omega(Z / Y) ; \mathbb{Z}_{p}^{\wedge}\right) \otimes Q$ is finite dimensional, we see that $H^{*}\left(Z / Y ; \mathbb{Z}_{p}^{\wedge}\right) \otimes Q$ is a finitely generated polynomial algebra, and hence we have

$$
\left.H^{*}\left(B Y ; \mathbb{Z}_{p}^{\wedge}\right) \otimes Q \cong\left(H^{*}\left(Z / Y ; Z_{p}^{\wedge}\right) \otimes Q\right) \otimes\left(H^{*} B Z ; Z_{p}^{\wedge}\right) \otimes Q\right)
$$

Thus we can find a homomorphism (left inverse) of polynomial algebras $r: H^{*}\left(B Y ; Z_{p}^{\wedge}\right) \otimes Q \rightarrow H^{*}\left(B Z ; Z_{p}^{\wedge}\right) \otimes Q$ with $r \cdot f^{*}=i d$. Consequently $r \cdot \tilde{f}^{*} \cdot i^{*}$ $=i d$, since $f \simeq i \cdot \tilde{f}$. Hence $i^{*}$ is injective.

We claim that $i^{*}$ is surjective and hence this homomorphism is bijective. It's enough to show that the composition $\varphi=i^{*} \cdot r \cdot \tilde{f}^{*}$ is bijective.

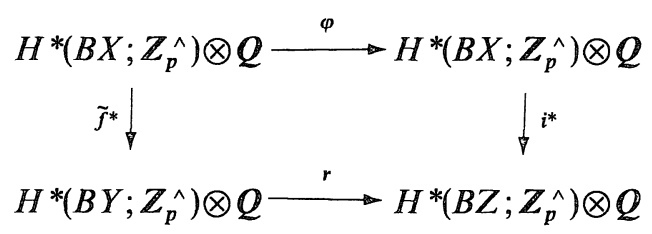

Since $i: B X \rightarrow B Z$ is a monomorphism and $i^{*}$ is injective, we see $\operatorname{rank}(X)=\operatorname{rank}(Z)$. Hence the $\mathrm{Krull}$ dimension of the image of $\varphi$ is equal to $\operatorname{rank}(X)$. Thus, at each degree, $\varphi$ is an injective linear self-map of a finite dimensional $Q_{p}^{\wedge}$-vector space, and therefore this linear map is bijective.

Consequently the monomorphism $i$ is a rational isomorphism. According to $[14$, Lemma $2.5(1)]$, we see that $B X$ is equivalent to $B Z$ under the map $i$.

Proof of Theorem 1. (1): We will show that if $\alpha \in f^{\perp}(B X, B Z)$, the composite map $B X \stackrel{\alpha}{\rightarrow} B Z \rightarrow B(Z / C(Z))$, say $q_{\alpha}$, is null homotopic.

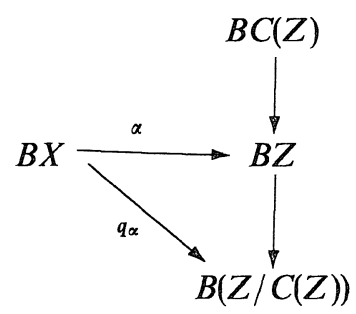


Using a result of Møller [14, Theorem 6.1], it's enough to prove that $q_{\alpha} \cdot \xi \simeq 0$ for any homomorphism $\xi: B Z / p^{n} \rightarrow B X$ and any $n \geq 1$. Since $\alpha \in f^{\perp}(B X, B Z)$, according to [8, Proposition 1.1], we see $\alpha \cdot \xi$ is contained in $f^{\perp}\left(B Z / p^{n}, B Z\right)$. So $f$ factors through $\operatorname{map}\left(B Z / p^{n}, B Z\right)_{\alpha \cdot \xi}$, which is the classifying space of the centralizer of $\alpha \cdot \xi$. A result of Dwyer-Wilkerson [3], [13, Theorem 5.1] shows that $\Omega \operatorname{map}\left(B Z / p^{n}, B Z\right)_{\alpha \cdot \xi}$ is a $p$-compact group and $e v: \operatorname{map}\left(B Z / p^{n}, B Z\right)_{\alpha \cdot \xi} \rightarrow B Z$ is a monomorphsim, since $Z / p^{n}$ is a $p$-compact toral group. If $\mu: B X \times B Y \rightarrow B Z$ is a pairing with restrictions (axes) $\left.\mu\right|_{B X} \simeq \alpha$ and $\left.\mu\right|_{B Y} \simeq f$, then the map $f: B Y \rightarrow B Z$ is expressed as the following composition:

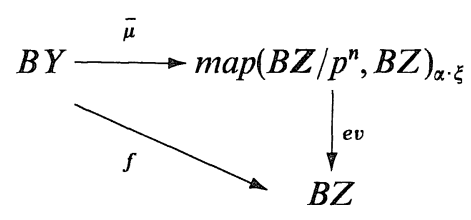

where $\bar{\mu}$ is induced by the adjoint map. In fact, for any $y \in B Y$, we see $e v \circ \bar{\mu}(y)=\bar{\mu}(y)(*)=\mu(\xi(*), y) \simeq f(y)$. Since $e v$ is a monomorphsim, by the assumption of $f$, Proposition 1 implies:

$$
\operatorname{map}\left(B Z / p^{n}, B Z\right)_{\alpha \cdot \xi} \simeq B Z
$$

Thus $\alpha \cdot \xi$ is central. Hence the map $q_{\alpha}: B X \rightarrow B(Z / C(Z))$ is null homotopic. Consequently, the map $\alpha: B X \rightarrow B Z$ factors through $B C(Z)$.

(2): Using [4, Theorem 9.3], one can show that the map of homotopy sets

$$
[B X, B C(Z)] \rightarrow[B X, B Z]
$$

is injective, since its kernel $[B X, Z / C(Z)]$ is trivial. The image of the map is included in $f^{\perp}(B X, B Z)$. We have just seen in part (1) that $[B X, B C(Z)]$ maps onto $f^{\perp}(B X, B Z)$. Consequently, $f^{\perp}(B X, B Z)=[B X, B C(Z)]$.

As seen in [8, Proposition 1.1], there is a strong relationship between pairing problems and mapping spaces. The following result shows that, for the homomorphism $f: B Y \rightarrow B Z$ in Theorem 1 , no $p$-compact groups find a difference between $B C(Z)$ and $\operatorname{map}(B Y, B Z)_{f}$. The proof uses the uniqueness of the pairing in our case. 
Corollary 1. Let $f: B Y \rightarrow B Z$ be as in Theorem 1. For any p-compact group $X$, the map of homotopy sets

$$
[B X, B C(Z)] \rightarrow\left[B X, \operatorname{map}(B Y, B Z)_{f}\right]
$$

is bijective, where the above map is induced by the canonical map

$$
B C(Z)=\operatorname{map}(B Z, B Z)_{i d} \rightarrow \operatorname{map}(B Y, B Z)_{f}
$$

Proof. First notice that there is a map

$$
\eta:\left[B X, \operatorname{map}(B Y, B Z)_{f}\right] \rightarrow f^{\perp}(B X, B Z)
$$

induced by adjoints. In fact, a map $B X \rightarrow \operatorname{map}(B Y, B Z)_{f}$ induces a pairing $B X \times B Y \rightarrow B Z$, and one of its axes is contained in $f^{\perp}(B X, B Z)$. Thus we get the following commutative diagram:

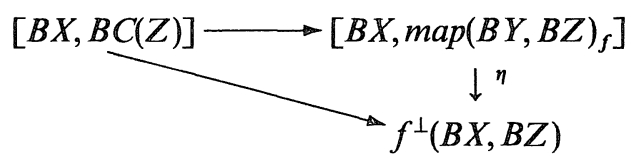

By [4, Lemma 5.3], for $\alpha \in f^{\perp}(B X, B Z)$, there is a unique pairing $\mu: B X \times B Y \rightarrow$ $B Z$ with $\left.\mu\right|_{B X} \simeq \alpha$ and $\left.\mu\right|_{B Y} \simeq f$. Hence $\eta$ is bijective. Theorem 1 shows $[B X, B C(Z)] \rightarrow f^{\perp}(B X, B Z)$ is bijective. Therefore the desired result holds.

Remark. This result seems to indicate that $\operatorname{map}(B Y, B Z)_{f}$ can be homotopy equivalent to $B C(Z)$ for such an $f$. For instance, if $\operatorname{map}(B Y, B Z)_{f}$ were shown to be a $p$-compact group, the statement would be true. When $f: B Y \rightarrow B Z$ is an epimorphism, as mentioned before, a result of Dwyer-Wilkerson [4, Lemma 10.3] implies $\operatorname{map}(B Y, B Z)_{f} \simeq B C(Z)$.

\section{§2. $H_{-}$-Structures on the Classifying Spaces}

In this section we will prove Theorem 2 using the following result, which is an easy consequence of Theorem 1.

Corollary 2. Suppose $X$ is a p-compact group. If $B X$ is an H-space, then $X$ is abelian.

Proof. Since $B X$ is an $H$-space, we see $\left(1_{B X}\right)^{\perp}(B X, B X)=[B X, B X]$. 
Because, if $m: B X \times B X \rightarrow B X$ is the $H$-multiplication, for any $\alpha \in[B X, B X]$, a pairing is given by the composite map $m \circ\left(\alpha \times 1_{B X}\right)$. Taking $\alpha=1_{B X}$ in Theorem 1, we see that the identity map of $B X$ factors through $B C(X)$. Proposition 1 implies $B X \simeq B C(X)$, and therefore $X$ is abelian.

Remark 1. A double loop space is homotopy commutative, and McGibbon [11] shows that $G_{p}$ is homotopy commutative if $p>2 n_{r}$ where $G$ is a simply-connected compact Lie group and $G \simeq{ }_{0} S^{2 n_{1}-1} \times \cdots \times S^{2 n_{r}-1}$ with $n_{1} \leq \cdots \leq n_{r}$. The twice deloopability or the existence of an $H$-structure on the classifying space is, however, far different from the homotopy commutativity, [12]. Corollary 2 implies $B G_{p}^{\wedge}$ is an H-space if and only if $G$ is a torus. We note here a theorem of Hubbuck [7]; Namely $T^{n}$ is the only nontrivial finite connected homotopy commutative $\mathrm{H}$-space.

Remark 2. Corollary 2 can be proved without using Theorem 1. We sketch the proof. Consider the fibration $B X_{0} \rightarrow B X \rightarrow B \pi_{0} X$ where $X_{0}$ denotes the identity component of $X$. Since $B X$ is an H-space, the connected $p$-compact group $X_{0}$ is a $p$-torus $T_{p}^{\wedge}$ and $\pi_{0} X$ is abelian. The map $B T_{p}^{\wedge}=B X_{0} \rightarrow B X$ is central, and $\left[B T_{p}^{\wedge}, B T_{p}^{\wedge}\right] \rightarrow\left[B T_{p}^{\wedge}, B X\right]$ is injective. Consequently the Weyl group $W(X)$ acts trivially on $B T_{p}^{\wedge}$. One can show that $B X \simeq B N T \simeq$ $B T_{p}^{\wedge} \times B \pi_{0} X$.

Proof of Theorem 2. First consider a connected finite loop space $X$. At any prime $p$, the $p$-completion $X_{p}^{\wedge}$ is a $p$-compact group, and $B X_{p}^{\wedge}$ is an H-space. Corollary 2 says that there is a torus $T^{n}$ such that $B X_{p}^{\wedge} \simeq\left(B T^{n}\right)_{p} \hat{\text {, }}$ where $n=\operatorname{rank}(X)$. Hence $B X \simeq B T^{n}$.

Next consider the general case so that we begin with the fibration $X_{0} \rightarrow X \rightarrow \pi_{0} X$ where $X_{0}$ denotes the identity component of $X$. Since $B X$ is an H-space, then $\pi_{0} X=\pi_{1} B X$ is abelian. Consequently, we have a fibration $B T^{n} \rightarrow B X \rightarrow B \pi_{0} X$. Notice [1] that this fibration is principal so that it preserved by the $p$-completion. So the loop space $\Omega B X_{p}^{\wedge}$ is a $p$-compact group. Corollary 2 says that there is a finite abelian $p$-group $\gamma_{p}$ such that $B X_{p}^{\wedge} \simeq\left(B T^{n}\right)_{p}^{\wedge} \times B \gamma_{p}$. We notice $B \gamma_{p}=\left(B \pi_{0} X\right)_{p}^{\wedge}$ so that $\pi_{0} X=\Pi_{p} \gamma_{p}$, since $\pi_{0} X$ is a finitely generated abelian group. Considering the fiber square, 


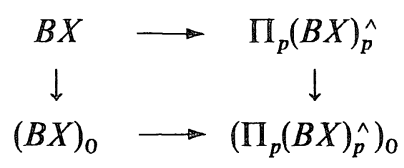

we see that the splitting of each $B X_{p}^{\wedge}$ induces a section for the fibration $B T^{n} \rightarrow B X \rightarrow B \pi_{0} X$. Since this fibration is principal, the classifying space $B X$ also splits. Consequently $B X \simeq B T^{n} \times B \pi_{0} X$.

If a compact Lie group $G$ is connected and the $p$-comletion of the classifying space $(B G)_{p}^{\wedge}$ is an H-space, then $G$ must be abelian. When $G$ is not connectd, however, the analogous result does not hold. A counter-example is given by a $p$-nilpotent group.

A finite group $\pi$ is called p-nilpotent, if the subgroup $v$ of $\pi$ generated by all elements of order prime to $p$ does not contain any $p$-torsion element. It is known that $\pi$ is the semidirect product $v \rtimes \pi_{p}$ where $\pi_{p}$ is the $p$-Sylow subgroup. Consequently, if $\pi_{p}$ is abelian, the $p$-completed space $(B \pi)_{p}^{\wedge} \simeq B \pi_{p}$ is an H-space (actually, an infinite loop space). Henn [6] provides a generalized definition of the $p$-nilpotence for compact Lie groups.

Theorem 3. Suppose $G$ is a compact Lie group and the p-completion of the classifying space $(B G)_{p}^{\wedge}$ is an $\mathrm{H}$-space. Then $G$ is the product of a torus $T$ and a finite p-nilpotent group $\sigma$ whose $p$-Sylow subgroup $\sigma_{p}$ is abelian, and hence $(B G)_{p}^{\wedge} \simeq(B T)_{p}^{\wedge} \times B \sigma_{p}$.

Proof. Suppose $P$ is a maximal $p$-toral subgroup of $G,[10]$. The $H$-structure on $(B G)_{p}$ induces a group homomorphism $P \times P \rightarrow P$ which makes $B P$ an $\mathrm{H}$-space, [5] and [16].

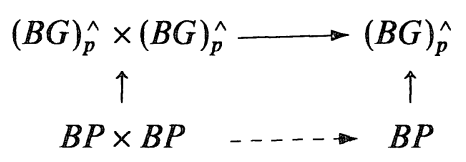

According to $[8$, Corollary 2.4], we see that $P$ is an abelian group. Let $N P$ be the normalizer of $P$ in $G$ and let $W=N P / P$. Since the maximal $p$-toral subgroup $P$ is abelian, the $\bmod p$ cohomology $H^{*}\left((B G)_{p}^{\wedge} ; F_{p}\right)$ is isomorphic to the ring of invariants $H^{*}\left(B P ; \mathbb{F}_{p}\right)^{W}=H^{*}\left(\left(B N P ; \mathbb{F}_{p}\right)\right.$ and therefore $(B G)_{p}$ 
$\simeq(B N P)_{p}^{\wedge}$. Consequently $(B N P)_{p}^{\wedge}$ has an $H$-structure:

$$
\mu:(B N P)_{p}^{\wedge} \times(B N P)_{p}^{\wedge} \rightarrow(B N P)_{p}^{\wedge}
$$

and we obtain the following diagram

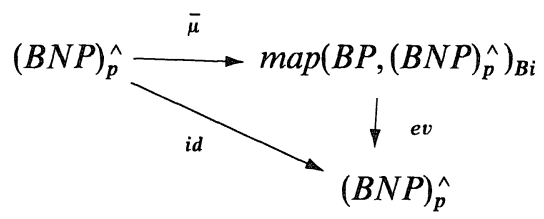

Notice [5] and [16] that $\operatorname{map}\left(B P,(B N P)_{p}^{\wedge}\right)_{B i} \simeq B P$, since the classifying space of the centralizer of $P$ in $N P=P \rtimes W$ is $p$-equivalent to $B P$. Consequently $(B N P)_{p}^{\wedge} \simeq B P$ and hence $(B G)_{p}^{\wedge} \simeq B P$. This implies that the compact Lie group $G$ is $p$-nilpotent in the sense of [6]. By [6, Proposition 1.3 and Theorem 2.5], we can show the desired result.

\section{References}

[ 1 ] Bousfield, A. and Kan, D., Homotopy limits, completions and localisations, LNM, 304 (1972).

[2] Broto, C. and Viruel, A., Homotopy Uniqueness of BPU (3), Preprint.

[3] Dwyer, W. G. and Wilkerson, C. W., Homotopy fixed-point methods for Lie groups and finite loop spaces, Ann. of Math., 139 (2), (1994), 395-442.

[4] The center of a p-compact group, The Čech centennial (Boston, MA, 1993), Contemp. Math., AMS, 181 (1995), 119-157.

[ 5] Dwyer, W. G. and Zabrodsky, A., Maps between classifying spaces, Proc. 1986 Barcelona conference, LNM 1298 (1987), 106-119.

[6] Henn, H. W., Cohomological p-nilpotence criteria for compact Lie groups, Théorie de l'homotopie, Astérisque, 191 (1990), 211-220.

[7] Hubbuck, J. R., On homotopy commutative $H$-spaces, Topology, 8 (1969), 119-126.

[8] Ishiguro, K., Classifying spaces and homotopy sets of axes of pairings, Proc. of AMS, 124 (1996), 3897-3903.

[9] Ishiguro, K. and Notbohm, D., Fibrations of classifying spaces, Trans. AMS, 343 (1) (1994), 391-415.

[10] Jackowski, S., McClure, J. E. and Oliver, B., Homotopy classification of self-maps of $B G$ via G-actions Part I and Part II, Ann. of Math., 135 (1992), 183-226, 227-270.

[11] McGibbon, C. A., Homotopy commutativity in localized group, Amer. J. Math., 106 (3) (1984), 665-687.

[12] Higher Forms of homotopy commutativity and finite loop spaces, Math. Z., 201 (1989), 363-374.

[13] Møller, J., Homotopy Lie groups, Bull. of AMS, 32 (4) (1995), 413-428.

[14] - Rational isomorphisms of p-compact groups, Topology, 35 (1) (1996), 201-225.

[15] Møller, J. and Notbohm, D., Centers and finite coverings of finite loop spaces, J. Reine Angew: Math., 456 (1994), 99-133.

[16] Notbohm, D., Maps between classifying spaces, Math. Z., 207 (1991), 153-168.

[17] Oda, N., The homotopy set of the axes of pairings, Canad. J. Math., 42 (1990), 856-868. 
[18] Viruel, A., Homotopy Uniqueness of $B G_{2}$, Preprint. 